\title{
Induction effects on ionospheric electric and magnetic fields
}

\author{
H. Vanhamäki, A. Viljanen, and O. Amm \\ Finnish Meteorological Institute, Space Research Unit, P.O.Box 503, FI-00101 Helsinki, Finland
}

Received: 15 November 2004 - Revised: 31 March 2005 - Accepted: 14 April 2005 - Published: 28 July 2005

\begin{abstract}
Rapid changes in the ionospheric current system give rise to induction currents in the conducting ground that can significantly contribute to magnetic and especially electric fields at the Earth's surface. Previous studies have concentrated on the surface fields, as they are important in, for example, interpreting magnetometer measurements or in the studies of the Earth's conductivity structure. In this paper we investigate the effects of induction fields at the ionospheric altitudes for several realistic ionospheric current models (Westward Travelling Surge, $\Omega$-band, Giant Pulsation). Our main conclusions are: 1) The secondary electric field caused by the Earth's induction is relatively small at the ionospheric altitude, at most $0.4 \mathrm{mV} / \mathrm{m}$ or a few percent of the total electric field; 2) The primary induced field due to ionospheric self-induction is locally important, $\sim$ a few $\mathrm{mV} / \mathrm{m}$, in some "hot spots", where the ionospheric conductivity is high and the total electric field is low. However, our approximate calculation only gives an upper estimate for the primary induced electric field; 3) The secondary magnetic field caused by the Earth's induction may significantly affect the magnetic measurements of low orbiting satellites. The secondary contribution from the Earth's currents is largest in the vertical component of the magnetic field, where it may be around $50 \%$ of the field caused by ionospheric currents.
\end{abstract}

Keywords. Geomagnetism and paleomagnetism (geomagnetic induction) - Ionosphere (electric fields and currents)

\section{Introduction}

In this paper we investigate the effects of induction on ionospheric electric and magnetic fields. Ionospheric currents may change very rapidly in response to magnetospheric driving, especially during magnetic storms and substorms. As the ionospheric current system and the accompanying primary magnetic field vary, a primary induced electric field is produced in accordance with Faraday's law of induction. In addition, if the Earth's conductivity is nonzero, the induced

Correspondence to: $\mathrm{H}$. Vanhamäki

(heikki.vanhamaki@fmi.fi) electric field drives currents in the Earth and these currents, in turn, produce the secondary electric and magnetic fields.

In the majority of studies where ionospheric processes are investigated, the ionospheric electric field is assumed to be curl-free, i.e. induction effects are ignored (e.g. Untiedt and Baumjohann, 1993). However, in some cases, for example, Alfvén wave reflection (Yoshikawa and Itonaga, 1996, 2000; Buchert, 1998), inductive effects may have a significant role. The inductive field is, of course, important in studies of induction in the Earth. In these studies the main attention is given to magnetic and/or electric fields at the Earth's surface, as these are needed, for example, when calculating geomagnetically induced currents (GIC) in man-made conductor systems (electric grids, pipelines, etc.; see, for example, Viljanen et al., 1999, 2004 and references therein) or in studies of the conductivity structure of the crust and upper mantle (e.g. Olsen, 1998, 1999; Constable and Constable, 2004).

In this paper our purpose is to investigate the relative magnitudes of the primary $\left(\boldsymbol{E}^{p}\right)$ and secondary $\left(\boldsymbol{E}^{s}\right)$ inductive fields with respect to the driving electric field $\left(\boldsymbol{E}_{0}\right)$ in different realistic situations. We also investigate the magnitude of the secondary magnetic field $\left(\boldsymbol{B}^{S}\right)$ produced by the Earth's currents as compared to the primary field $\left(\boldsymbol{B}^{p}\right)$ produced by the ionospheric currents at different altitudes and discuss the implications to satellite measurements. This is the first time that these effects are studied using realistic time-dependent three-dimensional models for high latitude ionospheric current systems.

Our nomenclature, already used above, concerning the different fields is as follows. The driving electric field $\boldsymbol{E}_{0}$ gives rise to the current density $\boldsymbol{J}_{0}=\overline{\bar{\Sigma}} \cdot \boldsymbol{E}_{0}$, where $\overline{\bar{\Sigma}}$ is the ionospheric conductance tensor. The models of ionospheric current systems that we use consist of $\boldsymbol{E}_{0}, \overline{\bar{\Sigma}}$ and $\boldsymbol{J}_{0} . \boldsymbol{E}_{0}$ is assumed to be caused by magnetospheric processes and mapped along the magnetic field lines to the ionosphere. In all the models we use $\nabla \times \boldsymbol{E}_{0}=0$, i.e. the input electric field does not include any induction effects. The currents $\boldsymbol{J}_{0}$ produce the primary magnetic field $\boldsymbol{B}^{p}$ and, if there is time dependence, the primary induced electric field $\boldsymbol{E}^{p}$, so that $\nabla \times \boldsymbol{E}^{p}=-\partial_{t} \boldsymbol{B}^{p}$. If the Earth's conductivity is nonzero, induced currents flowing in the Earth cause the secondary electric and magnetic fields, which also satisfy $\nabla \times \boldsymbol{E}^{s}=-\partial_{t} \boldsymbol{B}^{s}$. 
The total electric and magnetic fields in the ionosphere are $\boldsymbol{E}_{0}+\boldsymbol{E}^{p}+\boldsymbol{E}^{s}$ and $\boldsymbol{B}^{p}+\boldsymbol{B}^{s}$, respectively.

We assume the ionosphere to be a thin horizontal sheet $110 \mathrm{~km}$ above the ground, so that effects of spherical geometry and vertical variations within the ionosphere are ignored. Furthermore, the Earth's conductivity structure is assumed to be one-dimensional, so that the Earth consists of horizontal layers and each layer has a constant conductivity $\sigma$, permittivity $\epsilon$ and permeability $\mu\left(=\mu_{0}\right)$.

We represent the ionospheric current systems as superpositions of Cartesian Elementary Current Systems (CECS). Amm (1997) introduced two different CECS, one being curlfree and the other divergence-free. Any continuously derivable two-dimensional vector field can be presented as a sum of an infinite number of these elementary systems. The use of CECS simplifies the calculations, as analytical expressions for the electric and magnetic fields of these current systems exist.

The inductive response of the Earth is calculated using the complex image method (CIM) introduced to geophysical applications by Thomson and Weaver (1975). In CIM the layered Earth is replaced by a perfect conductor at a complex depth, and the secondary electric and magnetic fields produced by the currents flowing in the Earth can be calculated by the standard image principle. The depth of the perfect conductor $p$ can be calculated from the plane wave surface impedance $Z(\omega)$ as

$p=\frac{Z(\omega)}{i \omega \mu_{0}}$,

where $\omega$ is the angular frequency. Once the thicknesses and conductivities of the Earth layers are specified, the surface impedance $Z(\omega)$ can be calculated by using a recursion formula given, for example, by Wait (1981, pp. 52-53).

In most cases CIM gives very good approximations to the exact solutions and we believe it to be completely adequate for our purposes (see Thomson and Weaver, 1975; Boteler and Pirjola, 1998; Pirjola and Viljanen, 1998; Shepherd and Shubitidze, 2003, for discussions of accuracy). The neglection of horizontal variations in the Earth's conductivity may not always be a valid approximation, as large horizontal conductivity gradients exists, for example, between well conducting oceans and more resistive inland areas. However, in this study we are interested in the order of magnitude of the secondary fields and for this purpose the layered Earth assumption is adequate.

While the response of the conducting ground to external sources is described with a very good accuracy by CIM, our treatment of the ionospheric induction is more approximate. We calculate the primary and secondary induced electric fields $\boldsymbol{E}^{p}$ and $\boldsymbol{E}^{s}$ in the ionosphere using Eqs. (4, 6, 9 and 11). These equations give the fields in a vacuum, i.e. they do not take into account the response of the ionospheric plasma. In reality, the induced electric fields would drive currents in the ionosphere and these currents would cause their own second order induction phenomena, and so on. In this sense our treatment corresponds to terminating this iterative loop in the zeroth order. The higher order corrections would obviously decrease the total induced electric field, in accordance with Lenz's law, and therefore we obtain upper estimates for the induction effects. The induced currents in the ionosphere and associated field-aligned currents would also create their own magnetic fields both below and above the ionosphere, but we also neglect these effects.

The paper is organized so that in Sect. 2 we give the expression for the primary and secondary electromagnetic fields of the curl- and divergence-free elementary systems. In Sect. 3 we apply the combined CECS and CIM to different ionospheric model systems and compare the primary and secondary induced electric fields with the driving electric field of the models. Section 4 deals with the effect of Earth induction on satellite measurements of magnetic field variations at heights of a few hundred $\mathrm{km}$.

\section{Fields of elementary systems}

We use a coordinate system in which $x$ is northward, $y$ is eastward and $z$ is vertically down. The Earth's surface is the $x y$-plane and ionospheric currents are assumed to flow at height $h$ (i.e. $z=-h$ ). A more detailed derivation of the expressions for the primary and secondary electromagnetic fields of the divergence- and curl-free CECS is given in the Appendix; here we just give the final results.

The current density of the divergence-free CECS (Fig. 1), in cylindrical coordinates centered on the pole of the CECS, is given by Amm (1997) as (misprint in his formula corrected here)

$\boldsymbol{j}^{d f}=\frac{I_{0}}{2 \pi \rho} \delta(z+h) \hat{\boldsymbol{e}}_{\phi}$.

The magnitude of the elementary system is denoted by $I_{0}$, and $\delta$ is the Dirac delta function. This current system gives rise to a primary magnetic field

$$
\begin{aligned}
\boldsymbol{B}^{d f, p} & =\frac{\mu_{0} I_{0}}{4 \pi \rho}\left(\left[1-\frac{|z+h|}{\sqrt{\rho^{2}+(z+h)^{2}}}\right] \operatorname{sign}(z+h) \hat{\boldsymbol{e}}_{\rho}+\right. \\
& \left.+\frac{\rho}{\sqrt{\rho^{2}+(z+h)^{2}}} \hat{\boldsymbol{e}}_{z}\right)
\end{aligned}
$$

and a primary induced electric field (harmonic time dependence $e^{i \omega t}$ with the angular frequency $\omega$ is assumed)

$$
\boldsymbol{E}^{d f, p}=\frac{-i \omega \mu_{0} I_{0}}{4 \pi \rho}\left(\sqrt{\rho^{2}+(z+h)^{2}}-|z+h|\right) \hat{\boldsymbol{e}}_{\phi} .
$$

For a layered Earth model, the secondary fields produced by induced currents in the Earth can be calculated by using CIM. In this case the secondary fields of a divergence-free elementary system above the ground $(z \leq 0)$ are

$$
\boldsymbol{B}^{d f, s}=\frac{\mu_{0} I_{0}}{4 \pi \rho}\left(\left[1-\frac{h+2 p-z}{\sqrt{\rho^{2}+(h+2 p-z)^{2}}}\right] \hat{\boldsymbol{e}}_{\rho}-\right.
$$



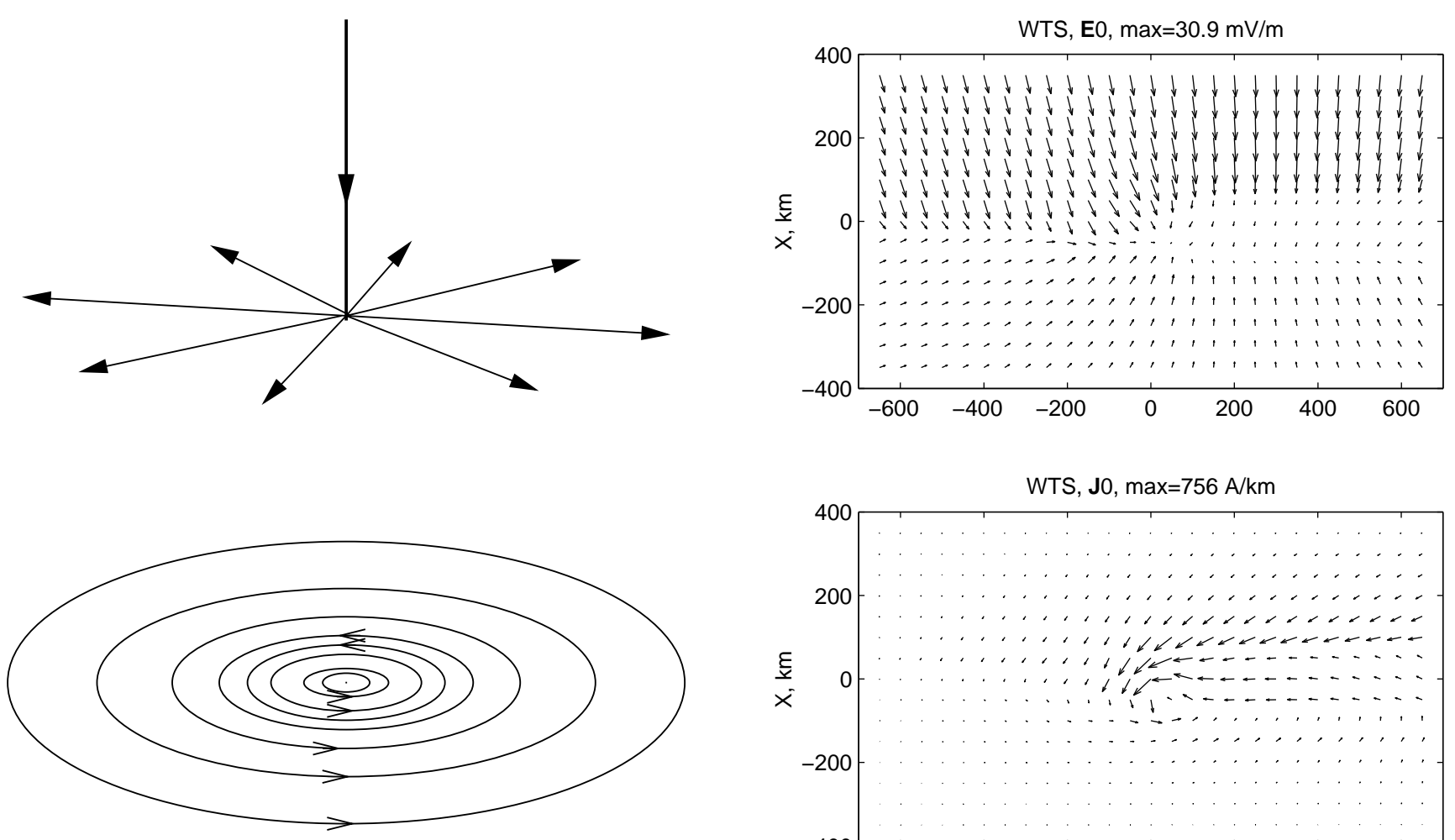

Fig. 1. The current density of the curl-free CECS (upper) and the divergence-free CECS (lower).

$$
\begin{gathered}
\left.-\frac{\rho}{\sqrt{\rho^{2}+(h+2 p-z)^{2}}} \hat{\boldsymbol{e}}_{z}\right) \\
\boldsymbol{E}^{d f, s}=\frac{i \omega \mu_{0} I_{0}}{4 \pi \rho}\left(\sqrt{\rho^{2}+(h+2 p-z)^{2}}-(h+2 p-z)\right) \hat{\boldsymbol{e}}_{\phi} .
\end{gathered}
$$

The depth of the perfect conductor $p$ is given by Eq. (1).

Similarly, for the curl-free CECS the current density is

$\boldsymbol{j}^{c f}=\frac{I_{0}}{2 \pi \rho} \delta(z+h) \hat{\boldsymbol{e}}_{\rho}+I_{0} \delta(x) \delta(y) U(-z-h) \hat{\boldsymbol{e}}_{z}$,

where $U$ is the Heaviside unit step function. The primary magnetic and electric fields are

$$
\begin{aligned}
\boldsymbol{B}^{c f, p} & =\frac{-\mu_{0} I_{0}}{2 \pi \rho} U(-z-h) \hat{\boldsymbol{e}}_{\phi} \\
\boldsymbol{E}^{c f, p} & =\frac{-i \omega \mu_{0} I_{0}}{4 \pi \rho}\left(\left[\sqrt{\rho^{2}+(z+h)^{2}}-|z+h|\right] \hat{\boldsymbol{e}}_{\rho}-\right. \\
& \left.-\rho \log \left(k_{0} \sqrt{\rho^{2}+(z+h)^{2}}+k_{0}(z+h)\right) \hat{\boldsymbol{e}}_{z}\right) .
\end{aligned}
$$

In the last equation $k_{0}=\omega / c$ is the vacuum wave number. In practise the z-component of the electric field is not important, because it (in good approximation) cancels due to the very high field-aligned conductivity in the ionosphere.

For a curl-free CECS CIM cannot be applied, but the secondary fields can be calculated in a different manner, as explained in the Appendix. For all geophysically reasonable

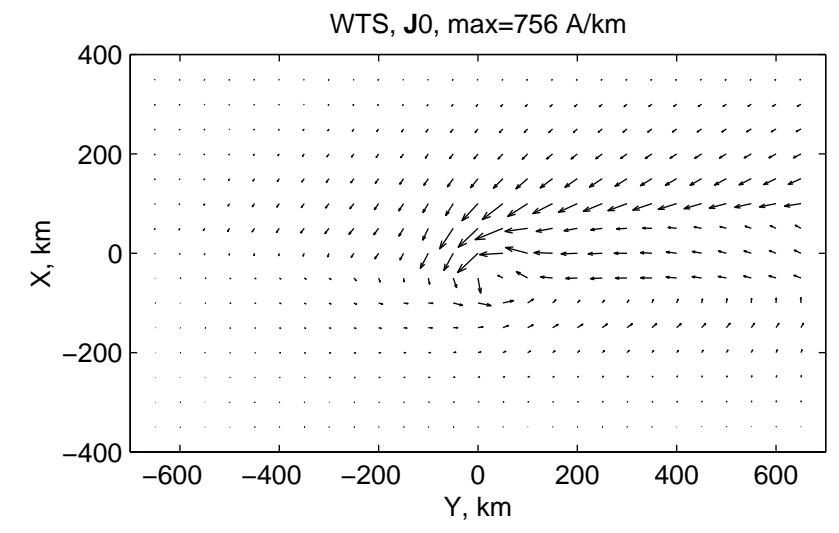

Fig. 2. The driving electric field and associated currents of the WTS model.

frequencies and conductivities the results can be approximated with negligible errors as

$\boldsymbol{B}^{c f, s}=0$

$$
\begin{aligned}
\boldsymbol{E}^{c f, s}= & \frac{i \omega \mu_{0} I_{0}}{4 \pi \rho}\left(\left[\sqrt{\rho^{2}+(h-z)^{2}}-(h-z)\right] \hat{\boldsymbol{e}}_{\rho}+\right. \\
& \left.+\left[\log \left(k_{0} \sqrt{\rho^{2}+(h-z)^{2}}+k_{0}(z-h)\right)\right] \hat{\boldsymbol{e}}_{z}\right) .
\end{aligned}
$$

Concerning the horizontal electric field, the Earth behaves as a perfect conductor, so that the horizontal part of the primary and secondary electric fields produced by curl-free CECS exactly cancel at the Earth's surface.

\section{Induced electric fields of different model systems}

In this section we will apply CIM for studying induction effects using several realistic models for ionospheric phenomena. These include Westward Travelling Surge (WTS), $\Omega$ band and Giant Pulsations. The driving electric fields and currents of the models are illustrated in Figs. 2-4. The WTS and $\Omega$-band models were composed from observational data by Amm (1995, 1996), who also gave the associated Hall and Pedersen conductivity distributions. The Giant Pulsation model is based on observations and analysis by Glassmeier (1980) and has been used by Viljanen et al. (1999). 

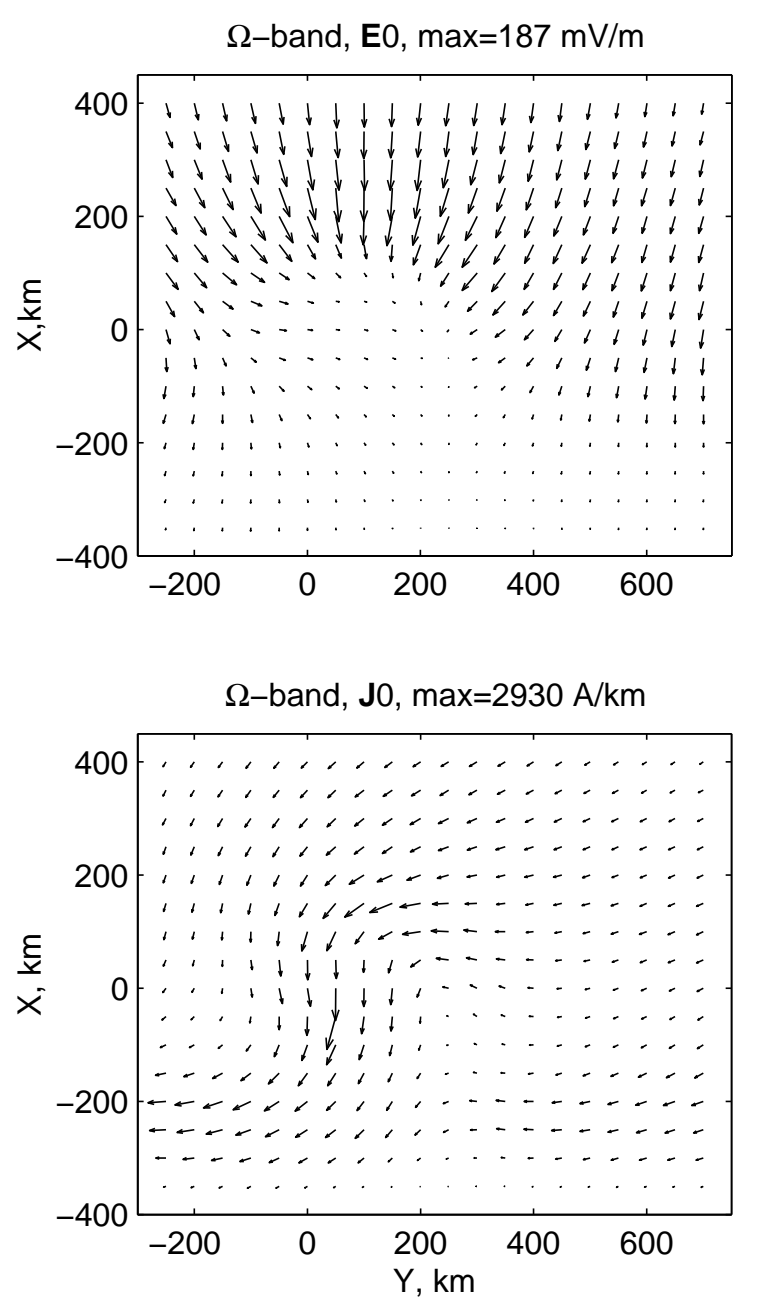

Fig. 3. The driving electric field and associated currents of the $\Omega$ band model.

Only the total current density is available for the Giant Pulsation model, so we assumed constant Hall and Pedersen conductances of 10 and $5 \mathrm{~S}$, respectively, when we calculated the electric field $\boldsymbol{E}_{0}$ for this model. These conductance values give a representative estimate for the driving electric field of the Giant Pulsations. However, in this case the resulting field $\boldsymbol{E}_{0}$ is not a pure potential field, but this does not affect the calculations.

\subsection{Calculation method}

The calculation of the primary and secondary fields $\boldsymbol{E}^{p}, \boldsymbol{B}^{p}$, $\boldsymbol{E}^{s}$ and $\boldsymbol{B}^{s}$ is done in 6 steps.

1. The horizontal current density of each model is represented by a sum of curl-and divergence-free CECSs placed at different locations in the model area.

2. The time variation $I_{0}(t)$ of the CECS amplitudes is calculated at each location. In the case of the WTS and $\Omega$ band models the time variation of the CECS amplitudes is calculated by moving the system in the east-west di-
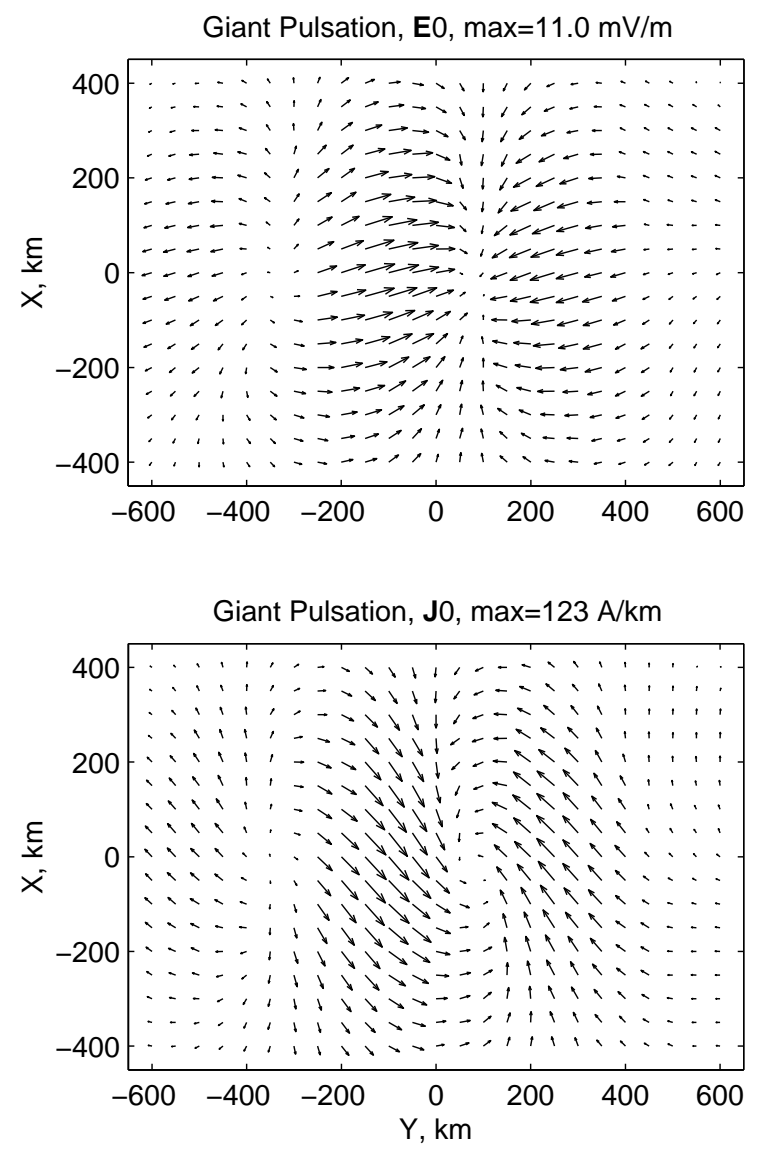

Fig. 4. Snapshot of the driving electric field and associated currents producing Giant Pulsations.

rection at a constant speed. The Giant Pulsation model already consists of several timesteps, one of which is illustrated in Fig. 4.

3. The time sequence of the CECS amplitudes $I_{0}(t)$ at each location is Fourier transformed to the frequency domain.

4. The primary and secondary fields for each frequency and CECS location are calculated using Eqs. (3-6 and $8-11)$.

5. The total fields are obtained by summing the contributions from the individual elementary systems.

6. Inverse Fourier transform gives the time domain fields.

In fact, all the primary fields and the secondary fields of the curl-free CECSs could be calculated analytically in the time domain, but the secondary fields of the divergence-free CECSs given in Eqs. (5-6) must be calculated in the frequency domain, as they depend on the complex depth $p(\omega)$ of Eq. (1). According to the properties of the inverse Fourier transform, this dependence on $p(\omega)$ also means that the inductive response of the conducting Earth is non-local in time. In other words, the secondary fields of the divergence-free 

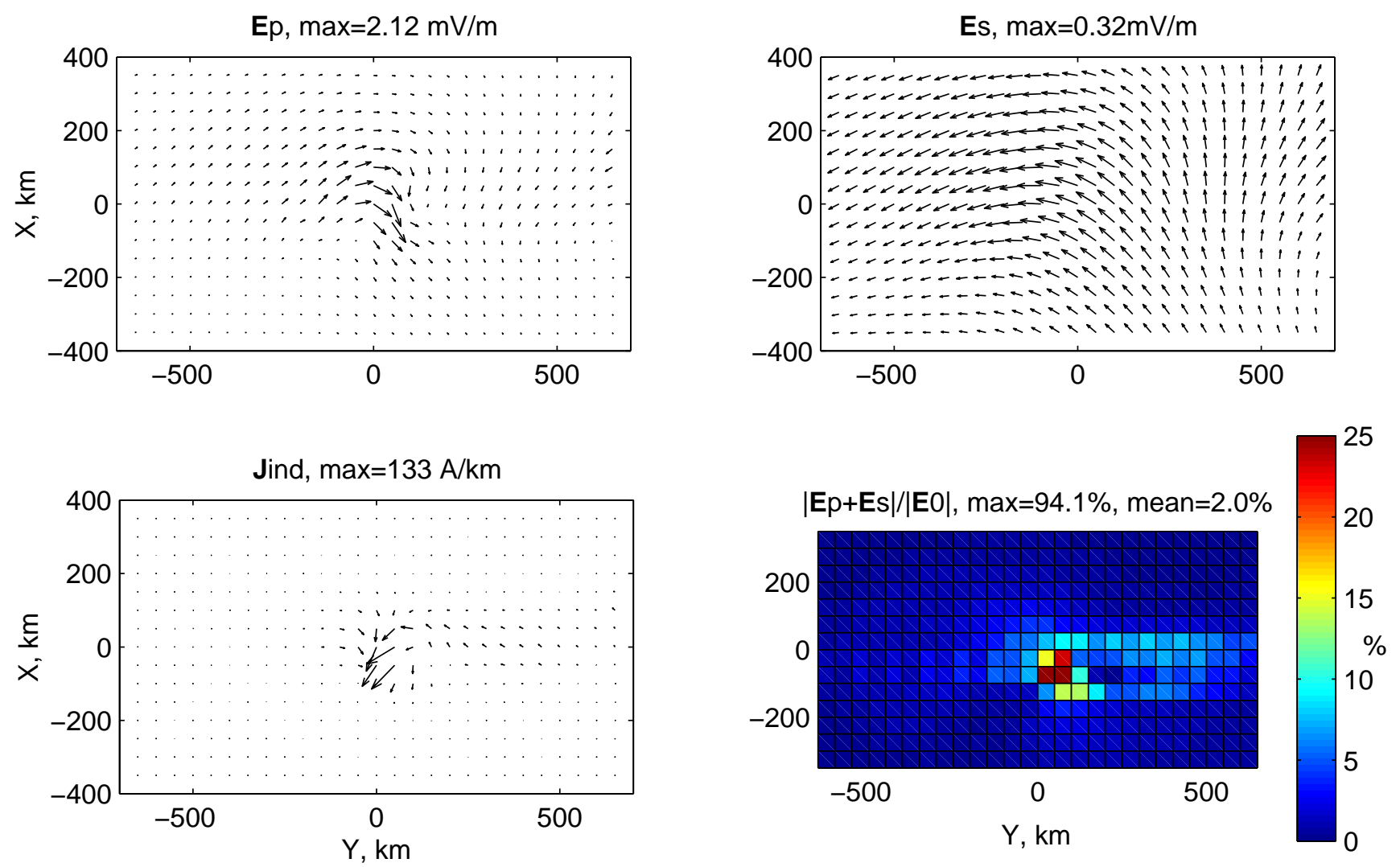

Fig. 5. Upper row: primary (left) and secondary (right) induced electric fields of the WTS model, note the different scales of the plots. Lower row: induced currents, i.e. currents driven by induced electric fields (left) and the ratio (primary+secondary induced electric field)/(driving electric field) (right). The color scale is limited to $25 \%$ while the maximum ratio is $94 \%$.

CECSs at time $t$ are not determined just from the amplitudes $I_{0}(t)$ at that same moment, but the fields are affected by the whole previous time development of $I_{0}\left(t^{\prime}\right), t^{\prime} \leq t$.

The primary magnetic field $\boldsymbol{B}^{p}$ describes the static field produced by the ionospheric currents and therefore it does not depend on the velocity of the current system or its oscillation frequency. The primary electric field $\boldsymbol{E}^{p}$ is caused by the time variations of the currents and according to Eqs. (4 and 9) the dependence on $\omega$ (and hence also on the velocity) is linear. The secondary fields of the divergencefree CECSs in Eqs. (5-6) depend on the complex depth $p(\omega)$ and therefore the detailed relationship between the secondary fields, the Earth's conductivity structure and the oscillation time of the Giant Pulsation or velocity of the WTS and $\Omega$ band models is complicated. However, it is clear that rapid time variations and large Earth conductivity result in larger induced currents within the Earth and also larger $\boldsymbol{B}^{s}$ and $\boldsymbol{E}^{s}$ above the Earth.

\subsection{Results}

Figures 5-8 show the resulting primary and secondary fields for the different models, together with comparison $\left|\boldsymbol{E}^{p}+\boldsymbol{E}^{s}\right| /\left|\boldsymbol{E}_{0}\right|$ against the driving electric field. Also, the induced ionospheric currents $\boldsymbol{J}^{\text {ind }}$ driven by $\boldsymbol{E}^{p}+\boldsymbol{E}^{s}$ are plotted. These currents were ignored in our calculations, so comparison of $\boldsymbol{J}^{\text {ind }}$ against $\boldsymbol{J}_{0}$ for each model system gives an estimate for the magnitude of correction terms these induced currents would produce. In these calculations we used a highly conducting "ocean" model in which the layer thicknesses and resistivities are $3,147, \infty \mathrm{km}$ (lowest layer is semi-infinite) and $0.25,100,1 \Omega m$, respectively. All the layers are assumed to have $\mu=\mu_{0}$ and $\epsilon=5 \epsilon_{0}$ (the exact value of $\epsilon$ is not important, as it appears only in the displacement current term that is negligible in the low frequency limit). The WTS system moves at a velocity of $10 \mathrm{~km} / \mathrm{s}$ westward and the $\Omega$-band at $2 \mathrm{~km} / \mathrm{s}$ eastward. These values are in the upper range of realistic speeds (e.g. Paschmann et al., 2002, Chapter 6). The oscillation time of the Giant Pulsation is about $100 \mathrm{~s}$.

It should be remembered that we calculate $\boldsymbol{E}^{p}$ and $\boldsymbol{E}^{s}$ as if the ionosphere were a vacuum, i.e. we ignore the currents driven by the induced electric fields. For this reason the results in Figs. 5-8 should be considered as upper limits for the induced electric fields, since the response of the ionospheric plasma should decrease the fields.

From Figs. 5, 7 and 8 it is obvious that in most cases the inductive electric fields are much smaller than the corresponding driving electric fields and that the secondary contribution to inductive fields is small compared to the primary part. 

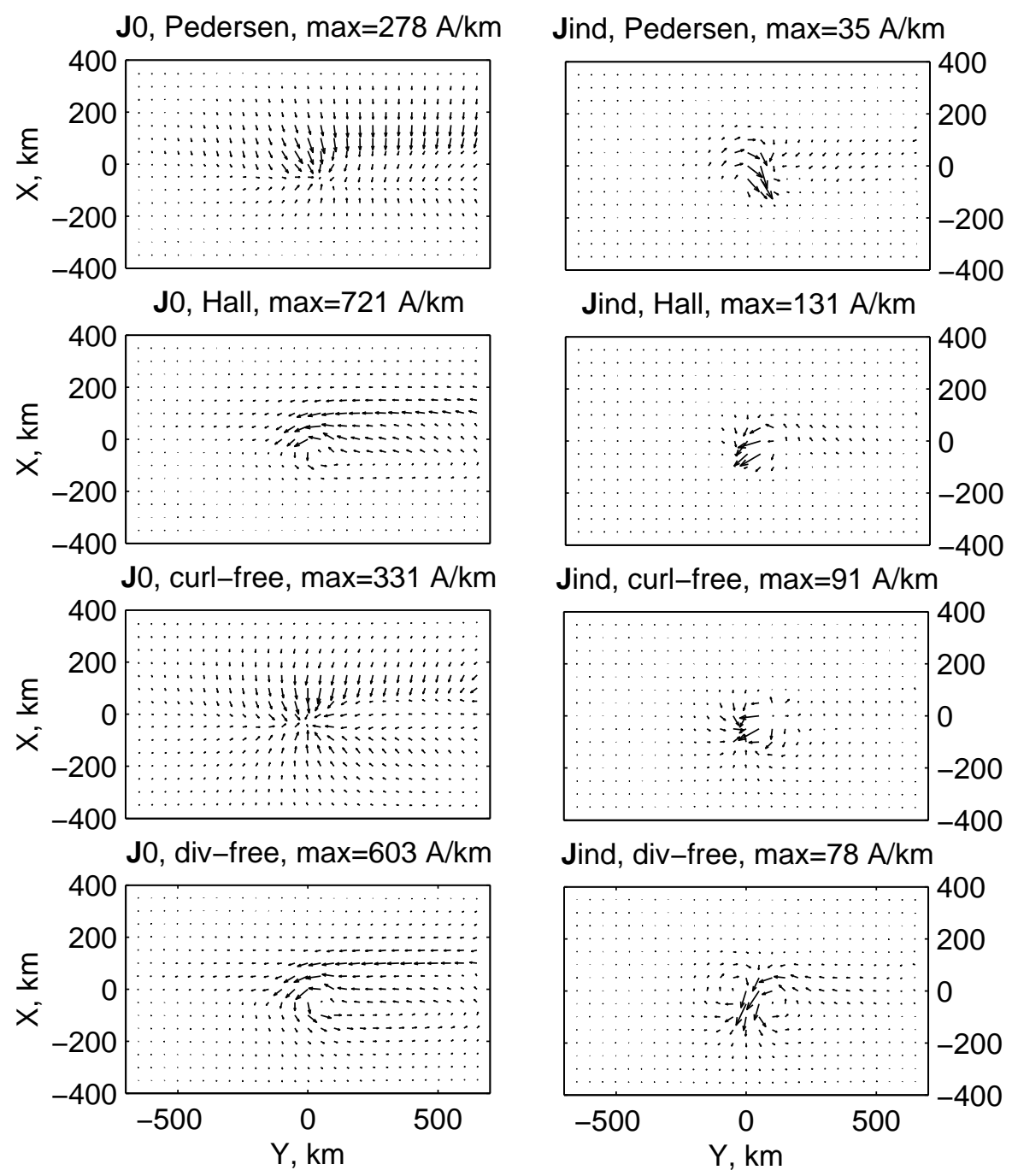

Fig. 6. The input current system (left side) and the induced current system (right side) of the WTS model decomposed into Pedersen, Hall, curl-free and divergense-free parts.

However, in some cases the primary inductive field may be large enough to significantly alter the driving electric field and therefore also the total current. These situations occur in regions of high conductivity, where the driving electric field is relatively weak. For example, in the "head" of the WTS model the relative magnitude of the total inductive field is $94 \%$ of the driving electric field, although the area involved is quite small. The average value of the ratio $\left|\boldsymbol{E}^{p}+\boldsymbol{E}^{s}\right| /\left|\boldsymbol{E}_{0}\right|$ over the whole WTS model area is just $2 \%$. A similar situation is visible in the Giant Pulsation case, where the maximum ratio is about $73 \%$ and the average value is about $6 \%$. For the $\Omega$-band model the maximum and average values are $13 \%$ and $1 \%$, respectively.

The induced currents, also plotted in Figs. 5-8, are in general much weaker than the original currents, as could be expected from electric fields results. However, the induced currents are not negligible. For example, in the WTS model the induced currents are locally quite large near the surge "head". This is the area where the largest FAC (field-aligned currents) flow in the original model. This means that the induction effects, although small in large scales, may significantly alter the magnetosphere-ionosphere coupling through modifying the FAC in this region. In Fig. 6 we have decomposed the original and induced currents of the WTS model into Pedersen, Hall, divergence-free and curl-free parts. The induced currents are associated with a pair of downward and upward FAC near the surge "head". In general, also in the other models the induced currents are not exactly opposite to the original currents, but nevertheless they tend to decrease the total FAC. We expect that proper treatment of the plasma response, i.e. the effects the induced currents cause, would tend to make the induced electric field and hence the currents more antiparallel to the original fields.

The secondary induced electric field is generally very smooth when compared to the primary one, as can be expected because of the larger distance to the image currents 

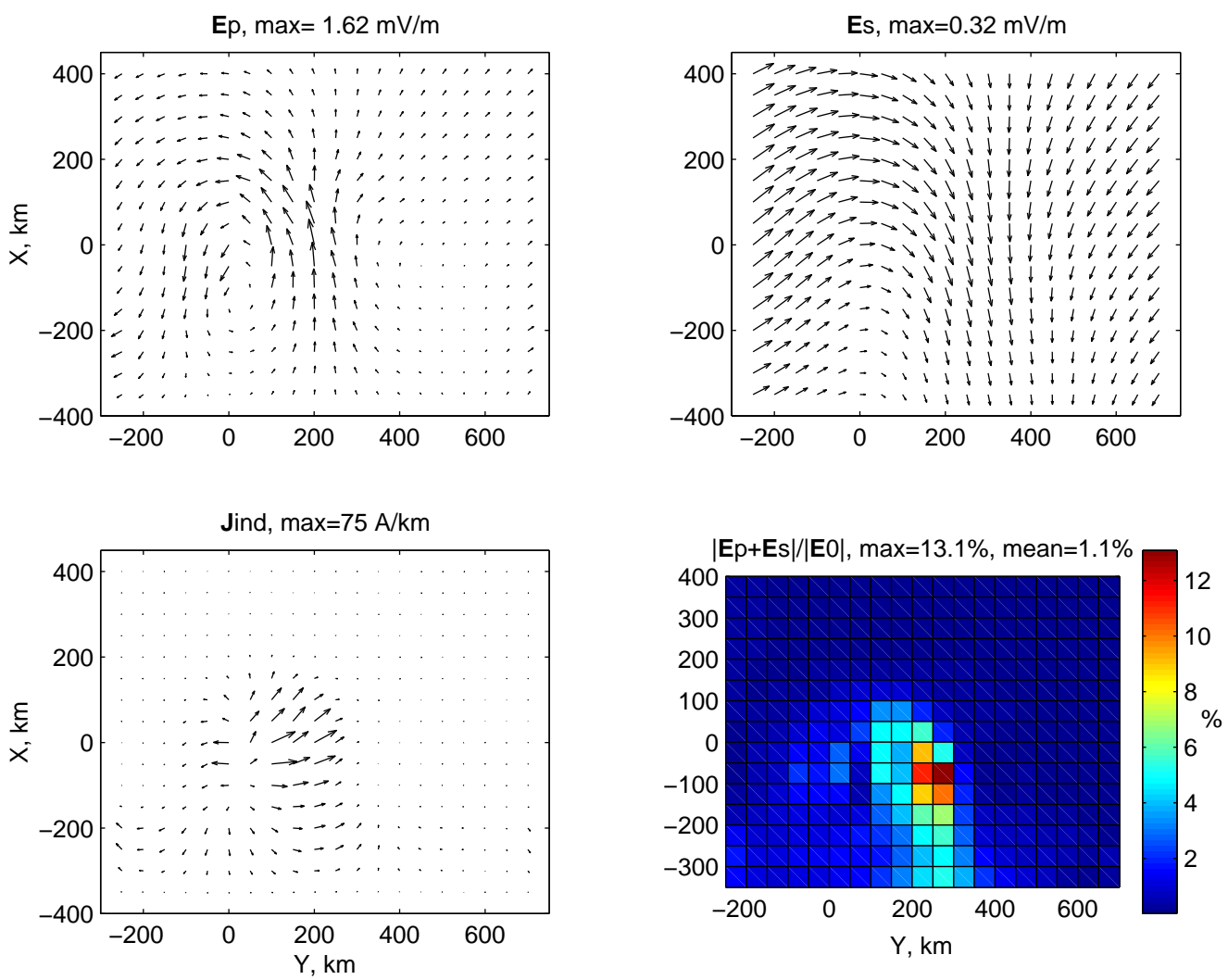

Fig. 7. Same as Fig. 5, but for the $\Omega$-band model. Note the different scales of the vector plots.
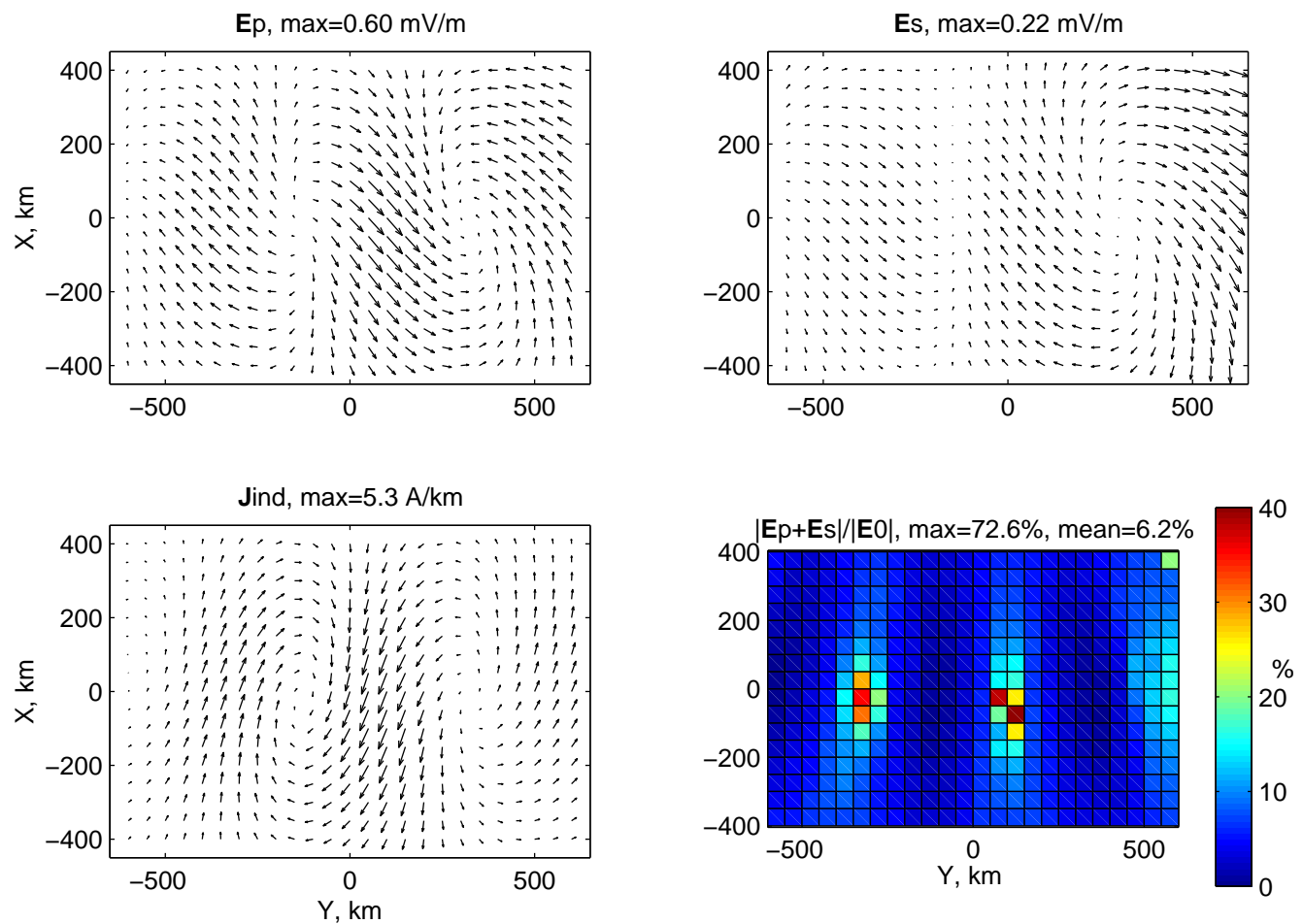

Fig. 8. Same as Fig. 5, but for the Giant Pulsation model. The color scale is limited to $40 \%$ while the maximum ratio is $73 \%$. Note the different scales of the vector plots. 

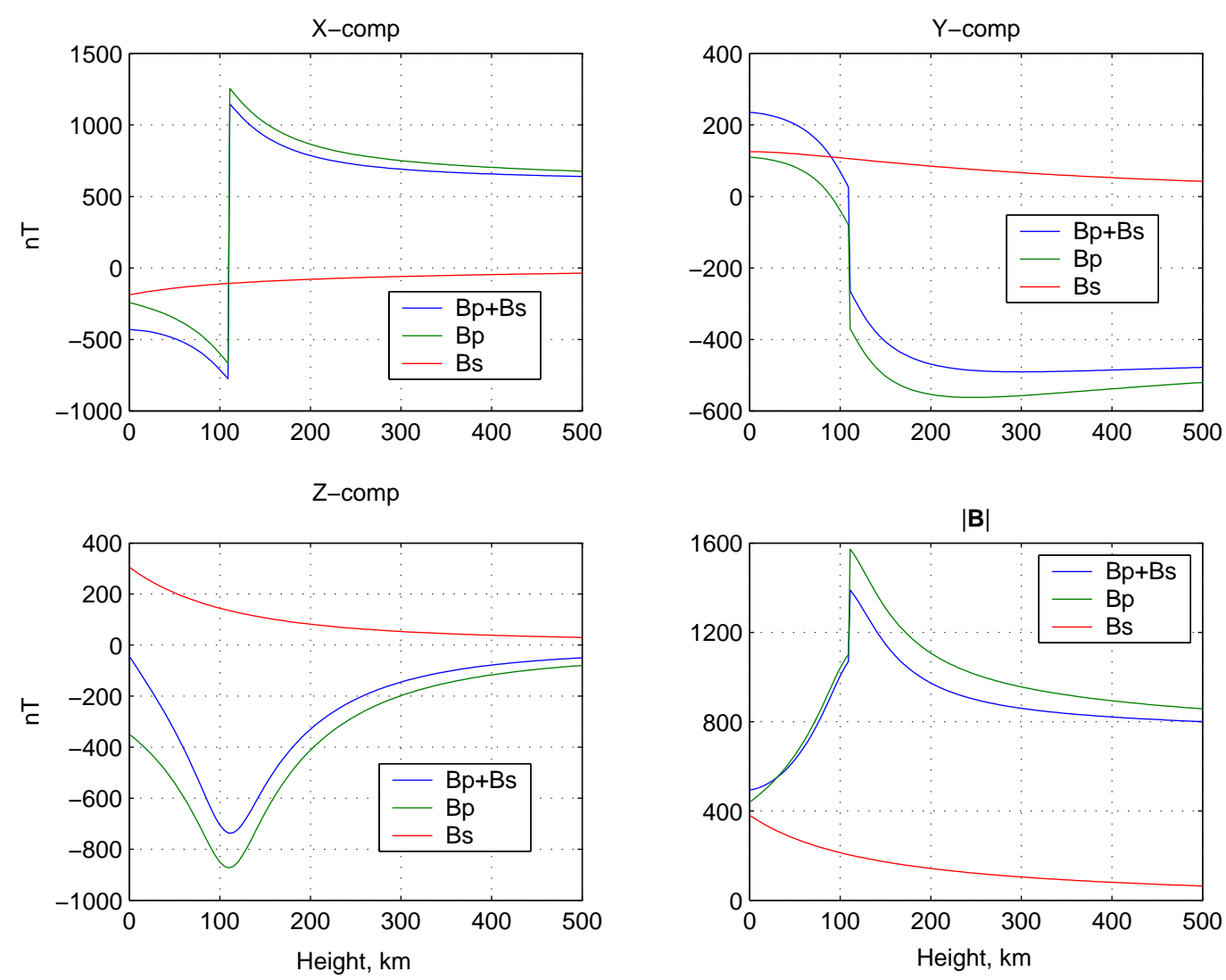

Fig. 9. Height profile of the primary and secondary magnetic fields at position $x=100 \mathrm{~km}, \mathrm{y}=200 \mathrm{~km}$ of the $\Omega$-band model.

within the Earth. In areas where the ratio $\left|\boldsymbol{E}^{p}+\boldsymbol{E}^{s}\right| /\left|\boldsymbol{E}_{0}\right|$ is highest, the ratio $\left|\boldsymbol{E}^{s}\right| /\left|\boldsymbol{E}^{p}\right|$ is usually lowest, as can be seen in Figs. 5, 7-8. The maximum values of the secondary field are about $15-35 \%$ of the primary field maximum values, and the secondary field is comparable to the primary one only in those areas where the inductive fields are very small.

We also made similar calculations for the Harang discontinuity (Amm, 1995) and Auroral Streamer (Amm et al., 1999) models. For the Harang discontinuity model the induced electric field is completely negligible, with the maximum ratio induced/driving field being $<2 \%$. This is expected because of the slow movement of the discontinuity. For the Auroral Streamer model the maximum inductive effect is also rather small but uniform, the maximum ratio is about $8 \%$, but the mean ratio is $4.5 \%$.

It seems that the secondary contribution to the total inductive electric field can be neglected, at least in the first approximation. If the Earth contribution is taken into account, representing the Earth with a perfect conductor should be a reasonable approximation in most cases. On the other hand, the primary inductive field may be locally very significant and it has to be taken into account when modelling dynamical ionospheric current systems. For example, in the case of Alfvén wave reflection (Yoshikawa and Itonaga, 1996, 2000; Buchert, 1998) the inductive response of the ionosphere may play a significant role.

\section{Magnetic field at satellite altitudes}

In this section we study the effect of the Earth's induction on magnetic fields at heights $300-500 \mathrm{~km}$ above ground, where satellites like CHAMP and Ørsted measure the geomagnetic field. We use the WTS and $\Omega$-band current models of Sect. 3, together with the "ocean" conductivity model.

The calculation method is the same as in the case of the induced electric fields, outlined in Sect. 3.1. Also in this case, the ionospheric response to $\boldsymbol{E}^{p}$ and $\boldsymbol{E}^{s}$ is ignored, i.e. the magnetic field associated with the ionospheric currents driven by these induced electric fields is not included in our calculations. The results in the previous section indicate that the ionospheric currents driven by $\boldsymbol{E}^{p}$ and $\boldsymbol{E}^{s}$ may produce large corrections to the magnetic field near the "head" of the WTS model, where the ratio $\left|\boldsymbol{E}^{p}+\boldsymbol{E}^{s}\right| /\left|\boldsymbol{E}_{0}\right|$ is large, but probably not in the $\Omega$-band model. In any case, our purpose here is to investigate the effect of the secondary magnetic field at the satellite altitudes. The secondary field is modelled very well by CIM and on average the currents driven by $\boldsymbol{E}^{p}$ and $\boldsymbol{E}^{s}$ should not change the ratio secondary/total magnetic field by more than a few percentage points.

The vertical profile of the primary and secondary magnetic fields at position $x=100 \mathrm{~km}, y=200 \mathrm{~km}$ of the $\Omega$-band model is shown in Fig. 9. The secondary field decreases relatively slowly with altitude, whereas the primary field peaks quite sharply at the current sheet. Above the current sheet the hor- 

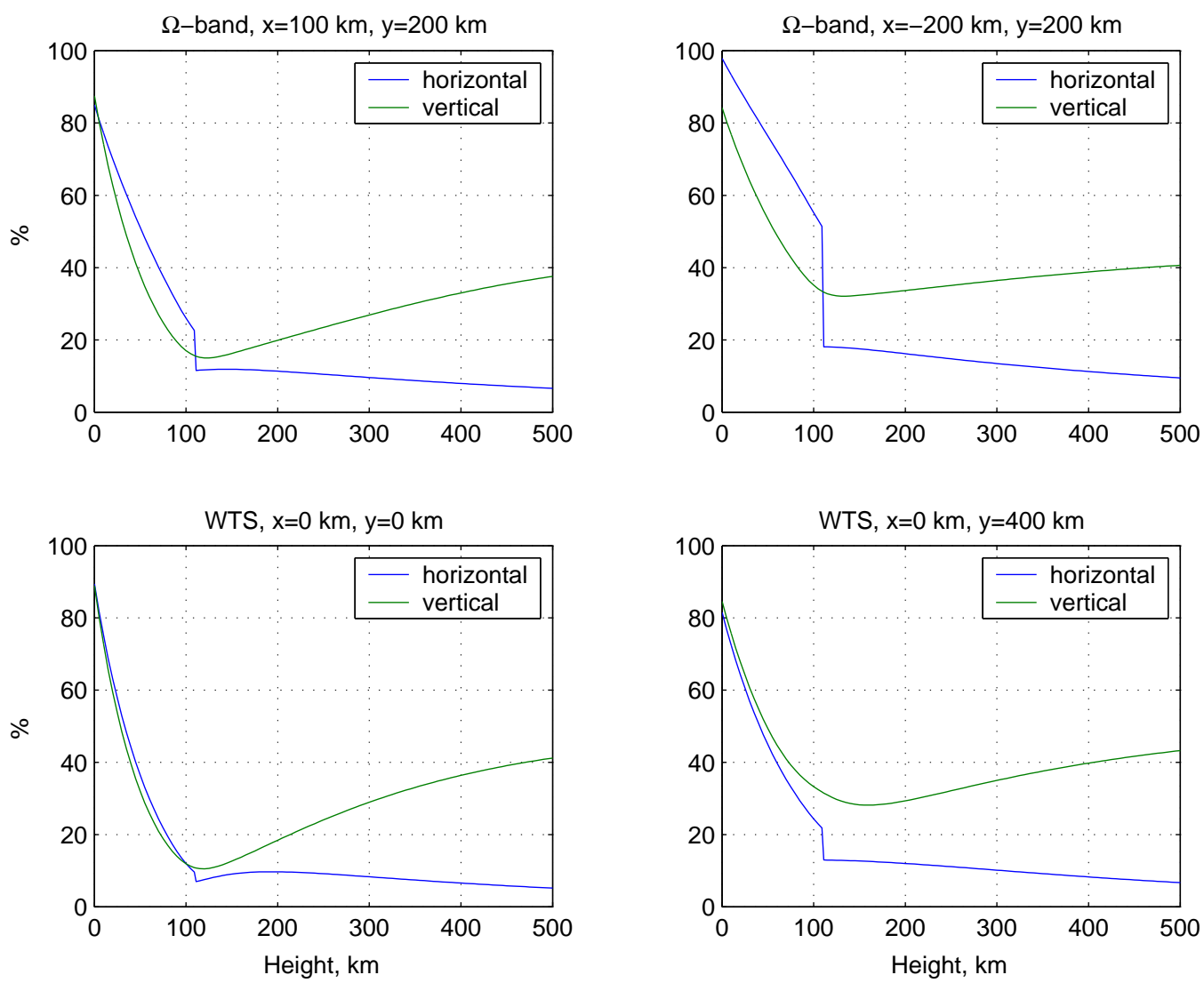

Fig. 10. Height profiles of the ratio secondary/primary magnetic field (horizontal and vertical components) at different locations of the WTS and $\Omega$-band models. The upper left panel corresponds to Fig. 9.

izontal part of the primary magnetic field is dominated by the field-aligned currents, and therefore it is essentially constant above $300 \mathrm{~km}$. The $z$-component of the magnetic field is affected only by the horizontal primary currents in the ionosphere and the secondary currents in the Earth. Therefore, at higher altitudes the contribution from the secondary currents should be more important in the $z$-component than in the horizontal components of the magnetic field.

Figure 10 shows height profiles of the ratio $\left|\boldsymbol{B}^{s}\right| /\left|\boldsymbol{B}^{p}\right|$ for the vertical and horizontal part of the magnetic field for two locations of the WTS and $\Omega$-band models. The upper left panel corresponds to Fig. 9. In these cases the fieldaligned currents decrease the ratio secondary/primary horizontal field to around $5-15 \%$ at altitudes above $250 \mathrm{~km}$. On the other hand, the vertical magnetic field is heavily affected by the secondary field, with the ratio secondary/primary field being about $20-50 \%$ between $250-500 \mathrm{~km}$.

The horizontal and vertical parts of the primary and secondary magnetic fields for the WTS and $\Omega$-band models at an altitude of $400 \mathrm{~km}$ are illustrated in Figs. 11-14. The secondary magnetic field is much smoother than the primary one and tends to be in the opposite direction. The horizontal part of the secondary field is much smaller than that of the primary field, but in the vertical fields the difference is smaller. It seems that in general the ratio secondary/primary field can be several tens of percent, even $50 \%$, for the vertical part, but only a few percent for the horizontal part.

The above examples indicate that inductive currents within the conductive Earth may have large effects on ionospheric magnetic field variations. We used a highly conducting Earth model representing ocean areas and rapidly changing ionospheric model systems, so the effect of the Earth's induction is in the upper range of realistic values in these examples. The Earth's effect should be taken into account when interpreting magnetic measurements made by low-orbit satellites. Of course, if the aim is to use satellite measurements in studies of the Earth conductivity structure, as, for example, recently done by Constable and Constable (2004), the secondary field is the important part. Olsen (1999) gives an extensive review of the different techniques used in these conductivity studies and discusses the obtained results. On the other hand, if one wants to study ionospheric current systems, one has to remove the secondary contribution, in order to obtain the right ionospheric currents. The simplest way of estimating the solid Earth contribution is to replace the Earth by a perfect conductor at a certain depth, as done, for example, by Olsen (1996). This may be completely adequate in many cases, although the depth of the perfect conductor should vary according to the large-scale conductivity properties of the underlying ground. 


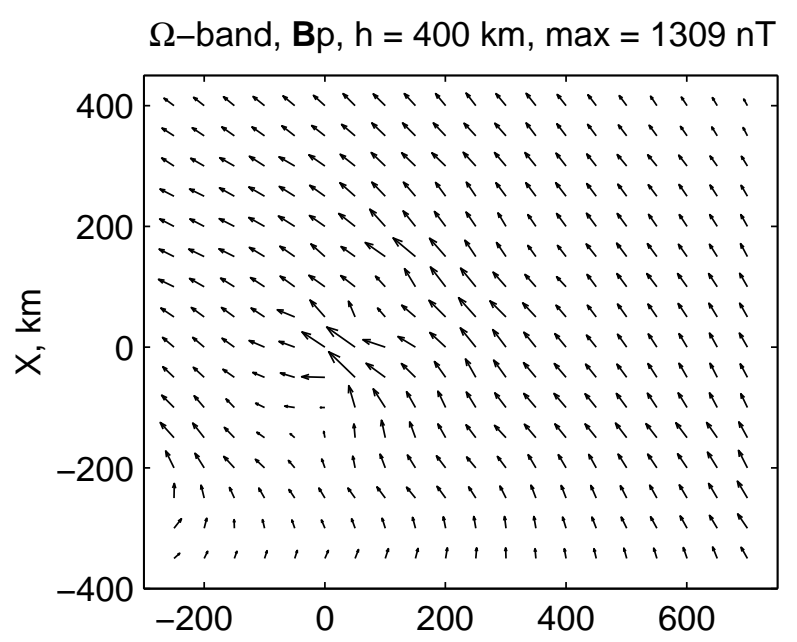

$\Omega$-band, Bs, h $=400 \mathrm{~km}, \max =77 \mathrm{nT}$

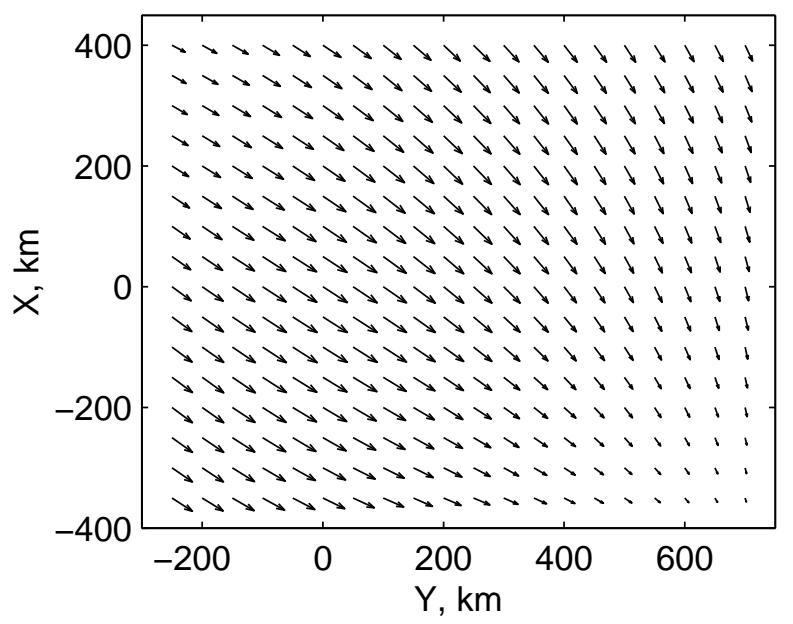

Fig. 11. The horizontal part of the primary and secondary magnetic fields of the $\Omega$-band model at an altitude of $400 \mathrm{~km}$. Note the different scales of the plots.

\section{Conclusions}

We have calculated the secondary electric and magnetic fields caused by the Earth's induction for several realistic models of ionospheric current systems. We have also estimated an upper limit of the electric field associated with ionospheric self-induction. Induction in the Earth was modelled accurately, assuming the Earth's conductivity does not vary in the horizontal directions, but the induced ionospheric fields were calculated as vacuum fields, that is, the ionospheric currents driven by the induced electric fields were ignored. Temporal variations of these currents would cause another induction loop, and so on. These corrections would tend to decrease the total induced electric field, in accordance with Lenz's law, so our calculations should give an upper estimate for the induced fields.

The calculated electric fields for different ionospheric models indicate that the secondary field $\boldsymbol{E}^{s}$ is very small
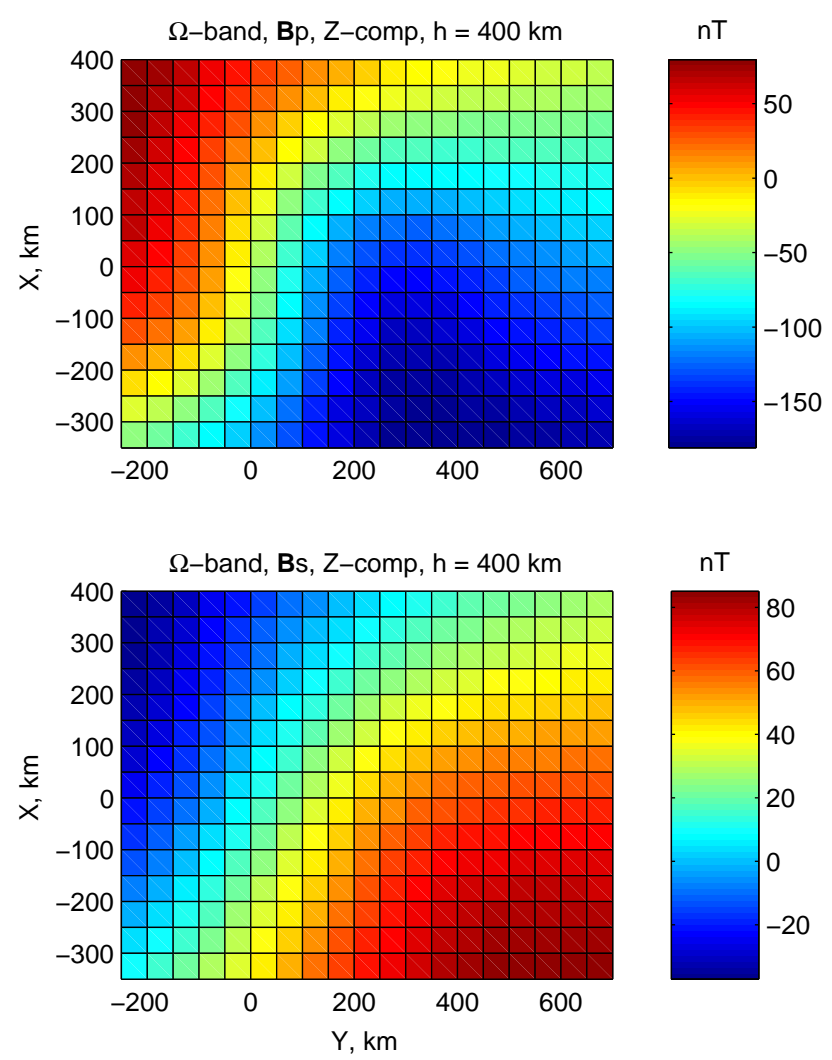

Fig. 12. The vertical part of the primary and secondary magnetic fields of the $\Omega$-band model at an altitude of $400 \mathrm{~km}$.

in most cases, both in absolute value and in comparison to the total electric field in the ionosphere. Thus, the Earth's induction can be neglected in studies of ionospheric electrodynamics, at least in most cases. If one wants to include the secondary electric field, replacing the Earth with a perfect conductor at some (real) depth should be a completely adequate approximation. At the Earth's surface the secondary electric field is of course important and must be taken into account, but at the ionospheric altitude the secondary field is small compared to the other electric fields.

The primary induced electric field $\boldsymbol{E}^{p}$, due to ionospheric induction, is larger than the secondary one and may be locally very important. For example, with the WTS model the primary field may be almost as large as the driving potential field $\boldsymbol{E}_{0}$ in some areas, as shown in Fig. 5. Similar "hot spots" are also present in the Giant Pulsation model and to a lesser extent also in the $\Omega$-band model, but not, for example, in the Harang discontinuity model. Our calculations give only upper estimates for the induced fields, as the ionospheric induction is not treated correctly. However, the results presented in this paper indicate that inductive phenomena may be important in ionospheric electrodynamics and a proper method for calculating the inductive fields in the ionosphere should be developed. A more precise treatment will be presented in a further paper. 

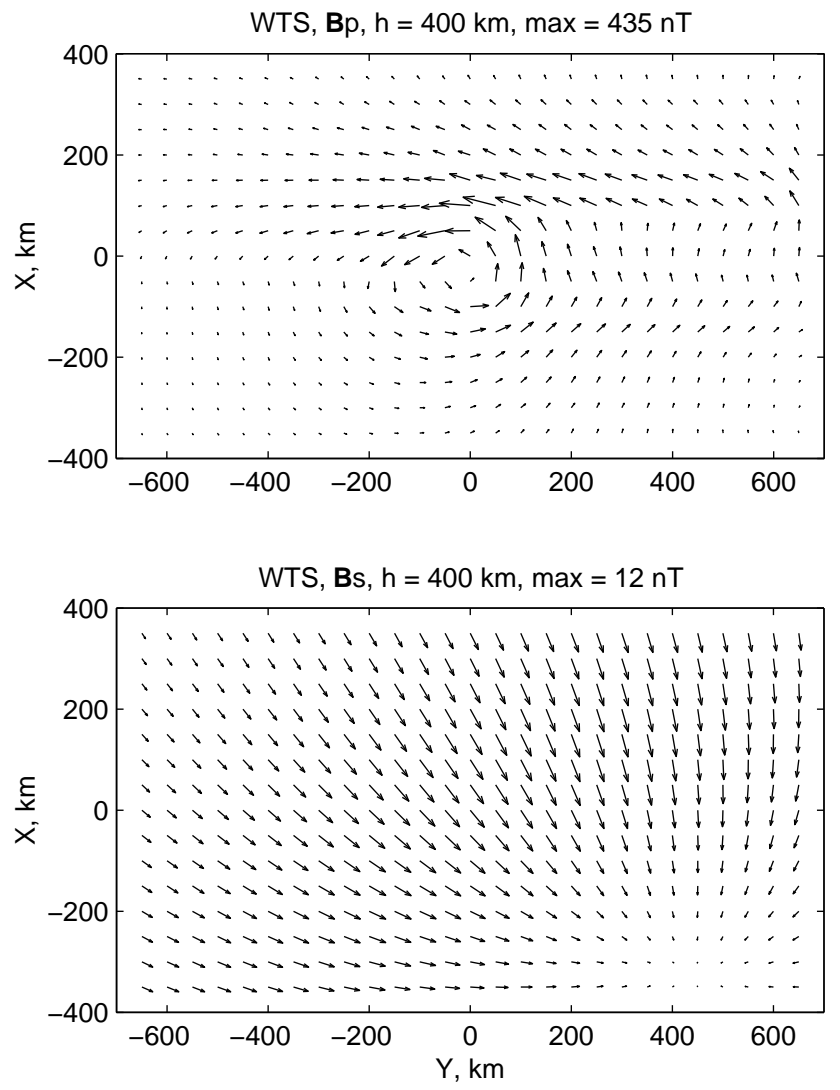

Fig. 13. Same as Fig. 11, but for the WTS model.

We also calculated the secondary magnetic field $\boldsymbol{B}^{s}$ at altitudes up to $500 \mathrm{~km}$, in order to estimate the effect of the Earth's induction on magnetic measurements made by loworbit satellites. In the horizontal part of the magnetic field the secondary contribution is rather small, less than about $15 \%$ of the primary part at altitudes above $300 \mathrm{~km}$. In the vertical part the ratio secondary/primary field may be much higher, $40-50 \%$ is not unusual. These results, calculated for the WTS and $\Omega$-band models, show that the Earth's induction must be taken into account when determining ionospheric currents from magnetic measurements made by loworbit satellites.

\section{Appendix A}

In this appendix we outline the calculation of the expressions in Eqs. (3-6 and 8-11) for the primary and secondary fields of the curl- and divergence-free elementary systems. Most of the results, together with detailed calculations, have already been presented by Pirjola and Viljanen (1998). However, they gave the fields only at the Earth's surface, so the results must be generalized. Some simplified expressions based on numerical calculations are also introduced.

Our starting point is Eq. (7) for the current density of the curl-free elementary system. Pirjola and Viljanen (1998)
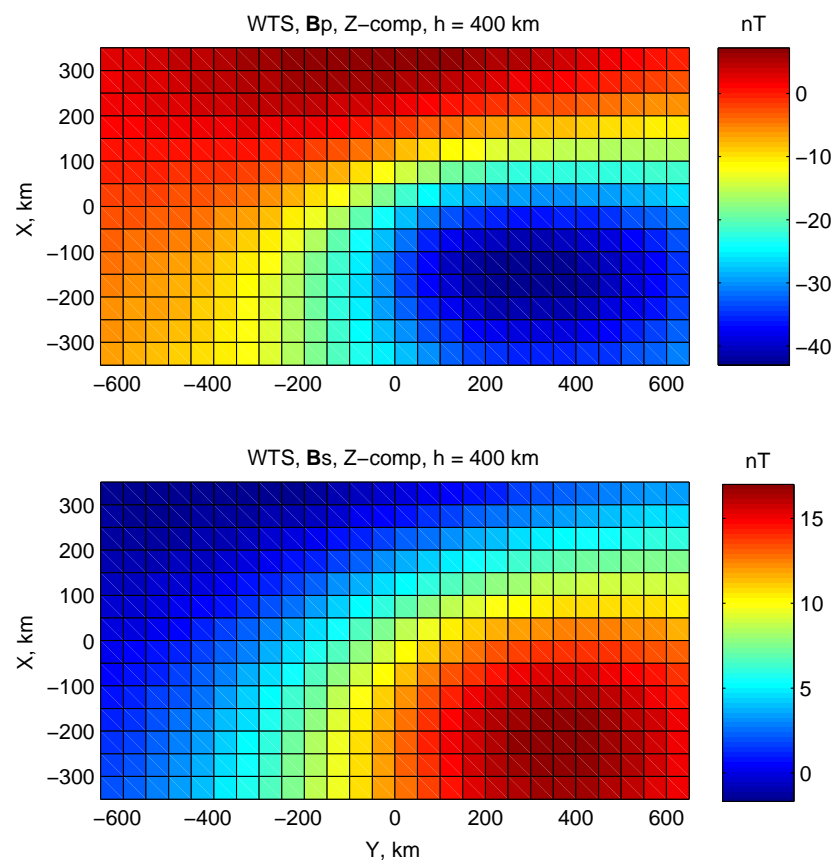

Fig. 14. Same as Fig. 12, but for the WTS model.

give the vector potential for this current system as (their Eqs. A17 and A22)

$$
\begin{aligned}
\boldsymbol{A}^{c f, p} & =\frac{\mu_{0} I_{0}}{4 \pi \rho}\left(\left[\sqrt{\rho^{2}+(z+h)^{2}}-|z+h|\right] \hat{\boldsymbol{e}}_{\rho}-\right. \\
& \left.-\rho \log \left(\sqrt{\rho^{2}+(z+h)^{2}}+(z+h)\right) \hat{\boldsymbol{e}}_{z}\right) .
\end{aligned}
$$

More detailed calculation including retardation effects for the line current would give the factor $k_{0}$ that is included inside the logarithm in Eq. (9) and other terms that vanish at least as fast as $\mathcal{O}\left(k_{0}\right)$ when $\omega \rightarrow 0$. The vector potential of the divergence-free CECS has only a horizontal part and it has exactly the same form as the horizontal part of the curlfree CECS's potential in Eq. (A1), except that is in the $\hat{\boldsymbol{e}}_{\phi^{-}}$ direction.

Using the above formulas for the vector potentials it is easy to verify the expressions for the primary fields given in Eqs. (3-4 and 8-9) by evaluating $\boldsymbol{B}=\nabla \times \boldsymbol{A}$, and $\boldsymbol{E}=-\partial_{t} \boldsymbol{A}$.

The CIM technique of Thomson and Weaver (1975) can be directly applied to the divergence-free CECS, as $\boldsymbol{j}^{d f}$ has only a horizontal part. Equations (5-6) clearly represent the fields of a mirror system placed at a complex depth $2 p+h$ below the surface, in accordance with having a perfect conductor at depth $p$.

The vertical line current in $\boldsymbol{j}^{c f}$ prevents the use of CIM for the secondary fields of the curl-free CECS. Pirjola and Viljanen (1998) calculated the secondary fields directly from Maxwell's equations for a homogenous Earth model, and we will apply these results. The relevant equations in Pirjola and 
Viljanen (1998) read (their Eqs. A2, A6, A9, A10, A11 and A29)

$$
\begin{aligned}
& B_{\phi}^{c f, s}=\int_{0}^{\infty} C(b) J_{1}(b \rho) e^{\kappa_{0} z} \mathrm{~d} b, \\
& E_{\rho}^{c f, s}=\frac{i \omega}{k_{0}^{2}} \int_{0}^{\infty} \kappa_{0} C(b) J_{1}(b \rho) e^{\kappa_{0} z} \mathrm{~d} b, \\
& E_{z}^{c f, s}=\frac{-i \omega}{k_{0}^{2}} \int_{0}^{\infty} b C(b) J_{0}(b \rho) e^{\kappa_{0} z} \mathrm{~d} b, \\
& C(b)=\frac{\mu_{0} I_{0}}{4 \pi} k_{0}^{2} k^{2} \frac{e^{-h b}}{b\left(k^{2} \kappa_{0}+k_{0}^{2} \kappa\right)}, \\
& k^{2}=\omega^{2} \mu \epsilon-i \omega \mu \sigma, \frac{-\pi}{4} \leq \arg (k) \leq 0, \\
& \kappa^{2}=b^{2}-k^{2}, \frac{-\pi}{2} \leq \arg (\kappa) \leq \frac{\pi}{2},
\end{aligned}
$$

These expressions are valid in the half space $z \leq 0 . \quad J_{0}$ and $J_{1}$ are the zeroth and first order Bessel functions, while $k_{0}$ and $\kappa_{0}$ are values of $k$ and $\kappa$ in the air, with parameters $\mu_{0}, \epsilon_{0}, \sigma=0$.

Numerical evaluation of the above expressions for the secondary fields of the curl-free CECS reveals that for all geophysically reasonable frequencies $\left(\omega=10 \ldots 10^{-4} s^{-1}\right)$ and conductivities $\left(\sigma=10 \ldots 10^{-5}(\Omega m)^{-1}\right)$ they can be approximated with the formulas given in Eqs. (10-11). Errors in these approximations are $<0.1 \%$, at least in the distance range $\rho \leq 500 \mathrm{~km}, 0 \leq z \leq 500 \mathrm{~km}$, where the fields are needed. Pirjola and Viljanen (1998) derived their analytical results for the homogenous conductivity case, but they also performed numerical calculations for a layered Earth conductivity model. These calculations confirmed that for a curl-free CECS the secondary magnetic field vanishes above the Earth and the secondary electric field can be calculated as if the Earth were a perfect conductor. Thus, Eqs. (10-11) can be also used with layered Earth conductivity models.

Acknowledgements. We thank R. Pirjola (at Finnish Meteorological Institute) for his comments on the manuscript. The work of H. Vanhamäki is supported by the Finnish Graduate School in Astronomy and Space Physics.

Topical Editor M. Lester thanks A. Yoshikawa and E. Woodfield for their help in evaluating this paper.

\section{References}

Amm, O.: Direct determination of the local ionospheric Hall conductance distribution from two-dimensional electric and magnetic field data: Application of the method using models of typical ionospheric electrodynamic situations, J. Geophys. Res., 100, 21 473-21 488, 1995.

Amm, O.: Improved electrodynamic modeling of an omega band and analysis of its current system, J. Geophys. Res., 101, 26772683, 1996.
Amm, O.: Ionospheric elementary current systems in spherical coordinates and their application, J. Geomag. Geoelect., 49, 947955, 1997.

Amm, O., Pajunpää, A., and Brandström, U.: Spatial distribution of conductances, currents, and field-aligned currents associated with a north-south auroral structure during a highly disturbed multiple-substorm period, Ann. Geophys., 17, 1385-1396, 1999, SRef-ID: 1432-0576/ag/1999-17-1385.

Boteler, D. and Pirjola, R.: The complex-image method for calculating the magnetic and electric fields produced at the surface of the Earth by the auroral electrojet, Geophys. J. Int., 132, 31-40, 1998.

Buchert, S.: Magneto-optical Kerr effect for a dissipative plasma, J. Plasma Physics, 59, 39-55, 1998.

Constable, S. and Constable, C.: Observing geomagnetic induction in magnetic satellite measurements and associated implications for mantle conductivity, Geochem. Geophys. Geosyst., 5Q01006, doi:10.1029/2003GC000634, 2004.

Glassmeier, K.-H.: Magnetometer Array Observations of a Giant Pulsation, J. Geophys., 48, 127-138, 1980.

Olsen, N.: A new tool for determining ionospheric currents from magnetic satellite data, Geophys. Res. Lett., 23, 3635-3638, 1996.

Olsen, N.: The electrical conductivity of the mantle beneath Europe derived from $C$-responces from 3 to 720 h, Geophys. J. Int. 133, 298-308, 1998.

Olsen, N.: Induction studies with satellite data, Surveys in Geophysics, 20, 309-340, 1999.

Paschmann, G., Haaland, S., and Treumann, R.: Auroral Plasma Physics, Space Sci. Rev., 103, 1-475, 2002.

Pirjola, R. and Viljanen, A.: Complex image method for calculating electric and magnetic fields produced by an auroral electrojet of finite length, Ann. Geophys., 16, 1434-1444, 1998,

SRef-ID: 1432-0576/ag/1998-16-1434.

Shepherd, S. and Shubitidze, F.: Method of auxiliary sources for calculating the magnetic and electric fields induced in a layered Earth, J. Atmos. Sol.-Terr. Phys., 65, 1151-1160, 2003.

Thomson, D. and Weaver, J.: The Complex Image Approximation for Induction in a Multilayered Earth, J. Geophys. Res., 80, 123$129,1975$.

Untiedt, J. and Baumjohann, W.: Studies of polar current systems using the IMS Scandinavian magnetometer array, Space Sci. Rev., 63, 245-390, 1993.

Viljanen, A., Amm, O., and Pirjola, R.: Modelling geomagnetically induced currents during different ionospheric situations, J. Geophys. Res., 104, 28 059-28 071, 1999.

Viljanen, A., Pulkkinen, A., and Amm, O., et al.: Fast computation of the geoelectric field using the method of elementary current systems and planar Earth models, Ann. Geophys., 22, 101-113, 2004,

SRef-ID: 1432-0576/ag/2004-22-101.

Wait, J.: Wave Propagation Theory, Pergamon Press New York, 1981.

Yoshikawa, A. and Itonaga, M.: Reflection of shear Alfvén waves at the ionosphere and the divergent Hall current, Geophys. Res. Lett., 23, 101-104, 1996.

Yoshikawa, A. and Itonaga, M.: The nature of reflection and mode conversion of MHD-waves in the inductive ionosphere: Multistep mode conversion between divergent and rotational electric fields, J. Geophys. Res., 105, 10 565-10 584, 2000. 